\title{
FAIMS Operation for Realistic Gas Flow Profile and Asymmetric Waveforms Including Electronic Noise and Ripple
}

\author{
Alexandre A. Shvartsburg, Keqi Tang, and Richard D. Smith \\ Biological Sciences Division, Environmental Molecular Sciences Laboratory, Pacific Northwest National \\ Laboratory, Richland, Washington, USA
}

\begin{abstract}
The use of field asymmetric waveform ion mobility spectrometry (FAIMS) has rapidly grown with the advent of commercial FAIMS systems coupled to mass spectrometry. However, many fundamental aspects of FAIMS remain obscure, hindering its technological improvement and expansion of analytical utility. Recently, we developed a comprehensive numerical simulation approach to FAIMS that can handle any device geometry and operating conditions. The formalism was originally set up in one dimension for a uniform gas flow and limited to ideal asymmetric voltage waveforms. Here we extend the model to account for a realistic gas flow velocity distribution in the analytical gap, axial ion diffusion, and waveform imperfections (e.g., noise and ripple). The nonuniformity of the gas flow velocity profile has only a minor effect, slightly improving resolution. Waveform perturbations are significant even at very low levels, in some cases $\sim 0.01 \%$ of the nominal voltage. These perturbations always improve resolution and decrease sensitivity, a trade-off controllable by variation of noise or ripple amplitude. This trade-off is physically inferior to that obtained by adjusting the gap width and/or asymmetric waveform frequency. However, the disadvantage is negligible when the perturbation period is much shorter than the residence time in FAIMS, and ripple adjustment appears to offer a practical method for modifying FAIMS resolution. (J Am Soc Mass Spectrom 2005, 16, 1447-1455) (C) 2005 American Society for Mass Spectrometry
\end{abstract}

S eparation of ions in the gas-phase using field asymmetric waveform ion mobility spectrometry (FAIMS) extends back two decades [1-7]. However, FAIMS was not widely used until its coupling to electrospray ionization mass spectrometry [8,9], which has expanded its utility to environmental [10-13] and biological [14-18] applications, in addition to traditional detection of airborne volatiles [19-23]. The recent advent of commercial FAIMS systems (Ionalytics Selectra $[17,24]$ and Sionex DMS $[23,25])$ is increasing the acceptance of FAIMS and diversifying its applications.

While FAIMS analyses have become increasingly common, the fundamentals remain relatively poorly understood [18]. Phenomenologically, ions are separated by the difference between mobilities at high and low electric fields $(E)$ (respectively $K_{\mathrm{H}}$ and $K_{\mathrm{L}}$ ) that in general differ as ion mobilities in gases depend on the field. Thus, FAIMS measures the mean derivative of mobility with respect to field $\langle\partial K / \partial E\rangle$ (over a certain range of $E$ ), in contrast to the conventional ion mobility spectrometry $[26,27]$ that determines absolute mobilities $(K)$. The $K(E)$ function can be expressed as a

Published online July 11, 2005

Address reprint requests to Dr. R. D. Smith, Biological Systems Analysis and Mass Spectrometry, Pacific Northwest National Laboratory, 3335 Q Ave., (K8-98), P.O. Box 999, Richland, WA 99352, USA. polynomial over even powers of $E / N$, where $N$ is the buffer gas number density [28, 29]:

$$
\begin{aligned}
K(E)= & K(0)\left(1+a(E / N)^{2}+b(E / N)^{4}\right. \\
& \left.+c(E / N)^{6}+\ldots\right)
\end{aligned}
$$

Mobilities tend to significantly deviate from zero-field values $K(0)$ at $E / N \geq \sim 30$ to $40 \mathrm{Td}$, which defines the lower limit for electric fields useful in FAIMS operation. The upper limit is given by the onset of electrical breakdown in gas, which depends on the gas identity and number density, and (to a lesser extent) the width and geometry of the gap between electrodes (the "analytical gap") in which the field is established [30]. Since the field in FAIMS is created by a time-dependent asymmetric waveform $V_{\mathrm{D}}(t)$, the breakdown threshold also depends on the oscillation frequency. Air and $\mathrm{N}_{2}$ at ambient conditions, the most common buffer gases, break down at $\sim 150-200 \mathrm{Td}$ while FAIMS typically operates at $\sim 70-100 \mathrm{Td}$. For $E / N<100 \mathrm{Td}$, truncating eq 1 after the $b(E / N)^{4}$ term is normally a good approximation $[28,29]$.

By the trend of $K(E)$ versus $E / N$ from zero to the maximum of the FAIMS operating range, ions (cations or anions) are classified into three types: A where $K(E)$ increases, B where $K(E)$ first increases and subsequently decreases, and $\mathrm{C}$ where $K(E)$ decreases. The key to 
rational design of FAIMS separations is a priori calculation of $K(E)$ for any ion/buffer gas pair. So far, this has been possible only when both the ion and buffer gases are monatomic, which creates an isotropic interaction potential that permits accurate scattering calculations $[31,32]$. For polyatomic ions, experiments have revealed that, in general, small ions including all monatomic species and nearly all small organic ions (including amino acids) belong to Type A, large and macromolecular ions such as proteins and polypeptides belong to Type C, and Type B is represented by relatively few intermediate-size species. These trends are for air or $\mathrm{N}_{2}$ buffer gases, in general the behavior depends on the gas. For example, $\mathrm{Cs}^{+}$that is A-type [33, 34] in $\mathrm{N}_{2}, \mathrm{O}_{2}$, or $\mathrm{CO}_{2}$ becomes C-type [35] in He and B-type [35] in Ne. Development of tools for quantitative prediction of $K(E)$ for polyatomic ions in any gas is a major challenge.

Experimentally, FAIMS separations proceed in an analytical gap where ions experience a field of $V_{\mathrm{D}}(t)$ with a superposed DC compensation voltage $(\mathrm{CV})$. Only ions with a specific form of $K(E)$ remain effectively stationary at any specific $C V$; all others migrate towards an electrode and are lost through neutralization. Thus, scanning CV yields a spectrum of ion species present. The earliest FAIMS analyzers featured two parallel planar electrodes [1]. The electric field in this configuration is spatially uniform, and ions freely diffuse in all directions under the influence of thermal diffusion and Coulomb repulsion. This diffusion may be suppressed in a field that is spatially nonuniform along its direction and has proper polarity (depending on the ion type), such as established in the annular gap between two coaxial cylinders where ions can be focused to the gap median [8]. This configuration (with a hemispherical cap) has been adopted in Ionalytics Selectra [17, 24]. In all commercial FAIMS systems, ions are carried through the gap by a gas stream. Alternatively, in a longitudinal field driven FAIMS ions are pulled along the gap by a constant electric field (created, for example, by segmented electrodes) [36, 37].

Analytical response in FAIMS is determined by a large number of instrumental parameters including the gap dimensions-shape (curvature), width $(g)$, and length $(L)$; operational variables - the peak amplitude (termed dispersion voltage, $\left.V_{\max }\right)$, frequency $\left(w_{\mathrm{c}}\right)$, and profile of $V_{\mathrm{D}}(t)$ waveform; and gas properties-composition, pressure, temperature $(T)$, and flow velocity $\left(v_{F}\right)$. The complex interplay of the factors controlling FAIMS resolution and sensitivity is not well understood, suggesting that FAIMS performance could be materially improved if all relevant trends could be mapped out.

Recently, we developed a numerical simulation of FAIMS analyzers [38] that covers planar, cylindrical, and spherical geometries. The overall concept is parallel to SIMION: a statistically representative ensemble of ions is propagated through the device, and properties of those passed are collected and aggregated. The underlying physical model incorporates ion diffusion (ac- counting for the high-field and anisotropic components) and space charge [38]. Calculations for resolution and sensitivity have previously been compared with measurements for a variety of systems across a wide range of ion sizes/types and FAIMS operational regimes and conditions [38, 39]. We have used this capability to probe the dependence of FAIMS response on several design and operational parameters, including $g, L, w_{\mathrm{c}}$, form of $V_{\mathrm{D}}(t)$, and the identity and flow velocity of buffer gas [39]. Elucidating the key trends as a function of these variables has allowed the FAIMS performance to be optimized depending on specific analytical priorities [39].

While that was the first realistic model for FAIMS operation under the stipulated conditions, those conditions were highly idealized. First, a uniform $v_{F}$ across the analytical gap was assumed. In reality, $v_{F}$ varies from zero at the gap walls to its maximum near the median, because of viscous friction. Also, ion motion (in the coordinate frame transposed with the gas flow) was limited to that in the direction of separation, i.e., across the gap. In reality, ions also diffuse along the gap. Second, FAIMS waveforms $V_{\mathrm{D}}(t)$ were assumed to be exactly as idealized. In a real experiment, any voltage contains electronic noise having a random component ("white noise") and often other contributions depending on the circuit design, such as a $60 \mathrm{~Hz}$ harmonic from the inductive AC power coupling. Background electronic noise is usually inconsequential in MS and IMS analyses, but strongly affects FAIMS as shown herein. Similarly, in addition to $\mathrm{CV}$ and $V_{\mathrm{D}}(t)$, FAIMS waveforms may contain a low-frequency symmetric component ("ripple"). That ripple may be a manifestation of noise (for example, at $60 \mathrm{~Hz}$ mentioned above) or be introduced deliberately to improve FAIMS resolution by effectively narrowing the gap [40].

Here we expand the model of FAIMS operation to account for true gas flow velocity distributions, axial ion diffusion, and real-life waveforms that comprise noise and ripple, and explore the effect of these phenomena on analytical response. The response depends on the ion current that controls the magnitude of space-charge effects, but all possibilities are encompassed by the limits of zero current (where those are absent) and saturated current (where the FAIMS charge capacity is filled and excess incoming ions are rapidly eliminated by Coulomb repulsion) [38, 39]. All simulations described are for pure buffer gases, but could be extended to gas mixtures via the formalism that accounts for non-Blanc effects on FAIMS separations [41].

\section{Advanced FAIMS Model}

Present simulations are built on the previously described procedure $[38,39]$. Briefly, an ion trajectory is tracked for the full residence time inside the gap $\left(t_{\text {res }}\right)$. Each ion experiences three phenomena: the electric field due to $\left\{V_{\mathrm{D}}(t)+C V\right\}$, high-field anisotropic diffusion in gas, and Coulomb repulsion from other ions. Various 
$V_{\mathrm{D}}(t)$ profiles were tested in experiment [8, 42-45] and theory [39], and rectangular waveforms with 2:1 ratio of high and low voltages $(f)$ provide the best performance $[1,39]$. However, rectangular waveforms with sufficiently high frequencies and amplitudes are difficult to generate [8]. Hence, nearly all FAIMS work has employed profiles that are easier to implement-the "bisinusoidal" and "clipped-sinusoidal" [21, 22, 44, 45] that produce very similar results [39]. In this study, we assume the bisinusoidal waveform (used in both Selectra and DMS) that is a sum of two harmonics with frequency ratio $f \approx 2$ :

$$
V_{\mathrm{D}}(t)=[f \sin w t+\sin (2 w t-\pi / 2)] V_{\max } /(f+1),
$$

where $w=2 \pi w_{\mathrm{c}}$. Ion transmission through the device under particular conditions is computed as a ratio of the number of exiting and initial trajectories. Evaluation of this ratio as a function of CV reveals the peak profile, with the width defined as full width at half-maximum (FWHM) and resolving power given as $\mathrm{CV}$ at peak apex divided by FWHM.

Simulations are performed for cylindrical FAIMS geometry and thus use the radial coordinate $r$. The planar geometry may be modeled by setting the radii of both internal $\left(R_{\text {in }}\right)$ and external $\left(R_{\text {out }}\right)$ electrodes to very large values, which reduces the curvature to zero.

\section{Gas Flow Velocity Distribution Across the Gap}

In fluid dynamics, gas flows around solid bodies are grouped into two regimes, laminar and turbulent [46]. Which would occur depends on the Reynolds number $(R e)$. In general, $R e=d v_{F} \rho / \mu$, where $\mu / \rho$ is gas kinematic viscosity $\left(\sim 1.7 \times 10^{-5} \mathrm{~m}^{2} / \mathrm{s}\right.$ for air or $\mathrm{N}_{2}$ at STP $)$ and $d$ is the characteristic body dimension [46]. For a planar gap or a slightly curved one in cylindrical FAIMS, $d=g$. Typical parameters are $\{g \sim 1.5-2 \mathrm{~mm}, v$ $\sim 0.1-0.5 \mathrm{~m} / \mathrm{s}\}$ for cylindrical analyzers such as Selectra and $\{g \sim 0.5 \mathrm{~mm}, v \sim 5-15 \mathrm{~m} / \mathrm{s}\}$ for planar devices such as DMS, yielding respectively $R e \sim 10-60$ and $R e \sim$ 150-400. These values are one-two orders of magnitude below the turbulence onset threshold $(R e \sim 3000)$ [46], hence all gas flows inside FAIMS are laminar. In other words, there is never a velocity component orthogonal to the flow direction that could carry ions across the gap and neutralize them at electrodes.

Laminar flows through pipes of any geometry have nonuniform distributions of $v_{F}$, with zero at the walls (here FAIMS electrodes) and maximum near the center (here the gap median) [46]. Thus $t_{\text {res }}$ (that are proportional to $1 / v_{F}$ ) are not fixed, but depend on ion trajectory through the gap: ions passing near the gap median will traverse FAIMS first, while those near either electrode will be delayed. The preferred location of a species in the gap depends on applied CV: ions pass mostly near the gap median at the CV corresponding to the spectral peak apex and closer to an electrode at CVs of peak edges. Thus residence times in FAIMS are

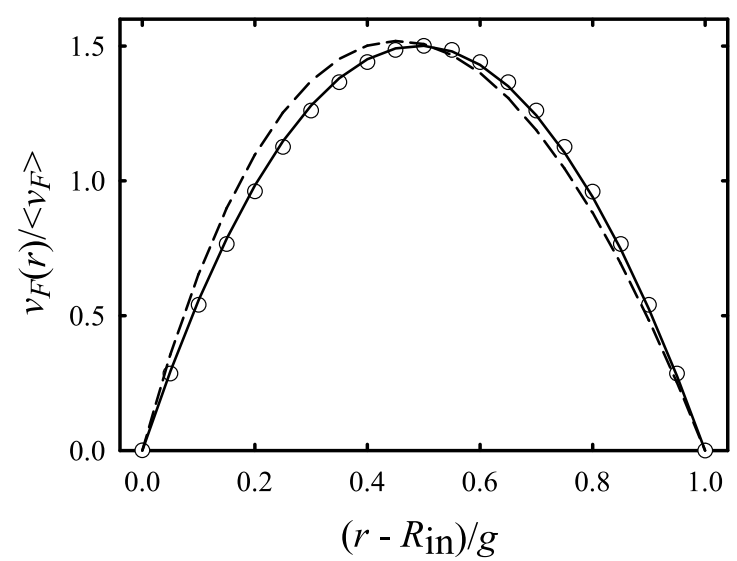

Figure 1. Calculated (steady-state) distributions of gas flow velocities across the FAIMS annular gap for $R_{\text {out }} / R_{\text {in }}=3$ (dashed line), 1.25 as in Selectra (solid line), and 1.0, i.e., the planar geometry (dots-the exact parabolic profile).

coupled to instrumental CV, which may affect the peak profile (experimental resolution). To introduce that into the model, we have evaluated $v_{F}(r)$ using standard fluid dynamics approaches [46]:

$$
\begin{gathered}
2\left\langle v_{F}\right\rangle\left(R_{\text {out }}^{2}-R_{\text {in }}^{2}\right)\left\{R_{\text {out }}^{2}-r^{2}\right. \\
v_{F}(r)=\frac{\left.+\left[\left(R_{\text {out }}^{2}-R_{\text {in }}^{2}\right) \ln \left(R_{\text {out }} / r\right)\right] / \ln \left(R_{\text {in }} / R_{\text {out }}\right)\right\}}{\left[R_{\text {out }}^{4}-R_{\text {in }}^{4}-\left(R_{\text {out }}^{2}-R_{\text {in }}^{2}\right)^{2} / \ln \left(R_{\text {out }} / R_{\text {in }}\right)\right]}
\end{gathered}
$$

where $\left\langle v_{F}\right\rangle$ is the average of $v_{F}(r)$. For a volume flow rate Q:

$$
\left\langle v_{F}\right\rangle=Q /\left[\pi\left(R_{\text {out }}^{2}-R_{\text {in }}^{2}\right)\right]
$$

The distribution [3] reduces to the textbook parabolic profile for a planar gap [46] when $g / R_{\text {in }} \rightarrow 0$, but is very close to it for all $g<R_{\text {in }}$ (Figure 1 ).

The eq 3 and its derivatives characterize the steadystate flow that develops over a finite (inlet) distance inside FAIMS. That distance was estimated as $L_{\mathrm{I}}=g R e$, amounting to $\sim 2-10 \mathrm{~cm}$ for Selectra and $\sim 10-30 \mathrm{~cm}$ for DMS [47]. Those values are comparable to or exceed lengths of those devices $(L \sim 1.5-3 \mathrm{~cm})$, and the initial uniform velocity distribution of free gas streams has been deemed to remain through the whole FAIMS analysis [47]. However, more accurately $L_{\mathrm{I}}=c g R e$, where $c \approx 0.03-0.05$, depending on the gap geometry [46]. This yields $L_{\mathrm{I}}$ of $0.05-0.5 \mathrm{~cm}$ for Selectra and $\sim 0.3-1.5 \mathrm{~cm}$ for DMS, indicating that FAIMS separations proceed largely in the steady-state regime, especially in Selectra. In any event, the $v_{f}(r)$ distribution in inlet region always lies between the uniform and the quasiparabolic one of eq 3, hence those two profiles define the limiting cases for FAIMS operation under any conditions.

Previously [38, 39], all ion trajectories were propagated for equal times of $t_{r e s}=L\left\langle v_{F}\right\rangle$. Now, at every 

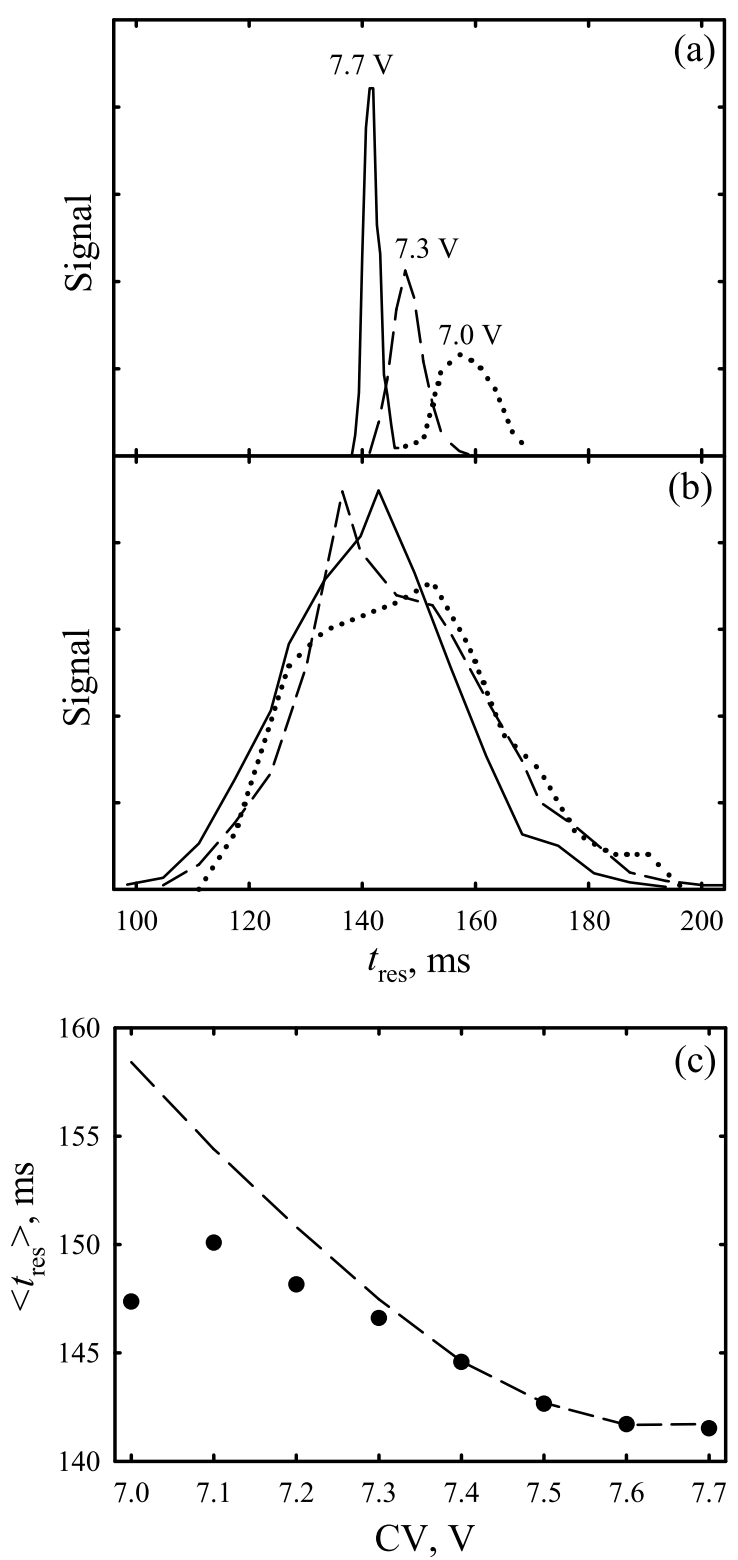

Figure 2. Ion residence times in FAIMS depend on CV. Distributions of $t_{\text {res }}$ across the (Leucine $\left.-\mathrm{H}\right)^{-}$peak are computed ignoring axial diffusion (a) and including it (b), for $\mathrm{CV}=7.7 \mathrm{~V}$ (peak apex, solid line), 7.3 V (peak side, dashed line), and 7.0 V (peak base, dotted line). Panel (c) shows mean $t_{\text {res }}$ as a function of $\mathrm{CV}$ for (a)-line and (b)-circles.

simulation step of duration $\Delta t$, for each $j$-th ion in the ensemble, we evaluate eq 3 depending on $r^{j}$, compute the axial displacement due to gas flow $\Delta x_{\mathrm{F}}^{\mathrm{j}}=v_{F}\left(r^{\mathrm{j}}\right) \Delta t$, and update the coordinate $x^{j}$. Each ion trajectory is propagated until $x^{j} \geq L$, i.e., the FAIMS exit is reached. Unless stated otherwise, simulations below are for $R_{\text {in }}$ $=7 \mathrm{~mm}, R_{\text {out }}=9 \mathrm{~mm}, w_{\mathrm{c}}=210 \mathrm{KHz}, V_{\max }=-3.3 \mathrm{kV}$, and $t_{\text {res }}=0.2 \mathrm{~s}$ (for the uniform $v_{F}$ ).

The effect of nonuniform $v_{F}(r)$ distribution on residence times is illustrated in Figure 2 using our exemplary $[38,39]$ (Leucine $-\mathrm{H})^{-}$species. At any $\mathrm{CV}$, there is a distribution of $t_{\text {res }}$ due to random ion motion sampling different parts of the gap. That distribution broadens and moves to longer times when $\mathrm{CVs}$ are shifted away from the peak apex (here $\sim 7.7 \mathrm{~V}$ ) in either direction: that pushes ions closer to electrodes where gas flows slower. The effect is modest because all ions that exit the gap have (on average) passed not too far from its median, and between the space sampled at $\mathrm{CV}$ $=7.7 \mathrm{~V}$ (peak apex) and $7.0 \mathrm{~V}$ (near baseline) $v_{F}(r)$ varies by $\sim 10-15 \%$ only (Figure $2 \mathrm{a}$ ). Ions that got close to electrodes where $v_{F}(r)$ falls drastically are lost anyway. The effect is more noticeable (though still small) when ions can pass closer to electrodes but survive. That happens in devices with higher curvature or for species with steeper $K(E)$ that both result in a stronger ion focusing, or when ion oscillations in the FAIMS cycle have a lower amplitude, for example at higher $w_{c}$. Importantly, mean $t_{\text {res }}$ are only $\sim 70 \%$ of the $0.2 \mathrm{~s}$ value derived for a uniform gas flow, and even the longest $t_{\text {res }}$ (at any CV) are significantly shorter than $0.2 \mathrm{~s}$.

As ions are continually neutralized on electrodes, a longer $t_{\text {res }}$ means lower transmission coefficient. This narrows CV spectral peaks thus improving resolution, but only slightly (by $\sim 4 \%$ in the example of Figure 3 ).

\section{Axial Ion Diffusion}

The original model $[38,39]$ was set up in single radial dimension, and the ion motion in gap plane (or axially and laterally for a cylindrical gap) other than with the coordinate frame of gas flow was ignored. In addition to being carried in the flow, ions randomly diffuse along the gap by Brownian motion, which affects the residence times. This diffusion is perpendicular to the field vector and thus is described by the transverse diffusional constant $D_{\mathrm{T}}$ :

$$
D_{\mathrm{T}}=k_{\mathrm{B}} T_{\mathrm{T}} K(E) / q
$$

where $k_{\mathrm{B}}$ is the Boltzmann constant, $q$ is ionic charge,

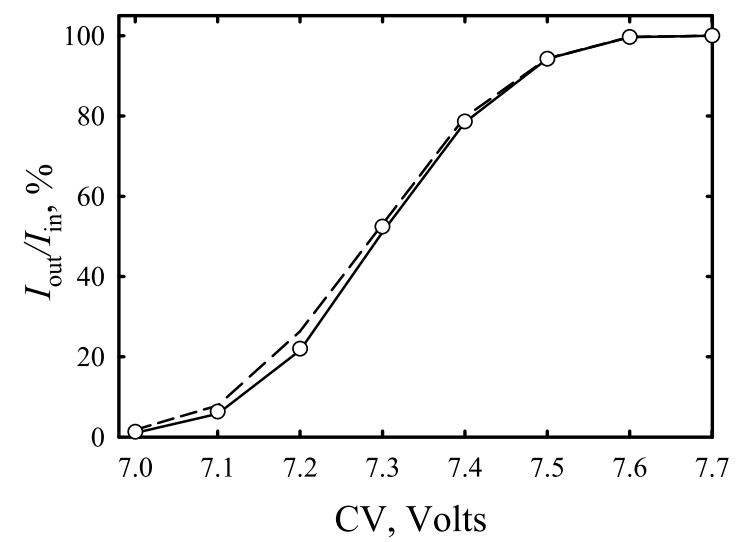

Figure 3. Simulated CV spectral profiles for scenarios of Figure 2 , ignoring axial diffusion for a uniform $v_{F}$ (dashed line) and steady-state $v_{F}(r)$ distribution (solid line), and including axial diffusion for the steady-state case (circles). Half of the peak is shown; the other half exhibits same behavior. The graph is for the zero ion current limit; the effect for saturated current is similar. 
and $T_{\mathrm{T}}$ is the "transverse temperature." Assuming an isotropic scattering,

$$
T_{\mathrm{T}}=T+[1-m /(2 m+M)] M v^{2}(r, t) /\left(3 k_{\mathrm{B}}\right)
$$

Here, $m$ and $M$ are the masses of respectively the ion and buffer gas molecule, and $v(r, t)$ is the instantaneous velocity of radial ion motion caused by electric field. Like the longitudinal diffusion constant, $D_{\mathrm{T}}$ is timedependent through $v(r, t)$ and is calculated for each ion at every simulation step. Then the axial displacement $\Delta x_{\mathrm{D}}^{\mathrm{j}}$ due to diffusion over $\Delta t$ is randomly selected (for each $j$ ) with probability given by the Gaussian distribution

$$
p\left(\Delta x_{\mathrm{D}}^{\mathrm{j}}\right)=\left(4 \pi D_{\mathrm{T}} \Delta t\right)^{-1 / 2} \exp \left[-\left(\Delta x_{\mathrm{D}}^{\mathrm{j}}\right)^{2} /\left(4 D_{\mathrm{T}} \Delta t\right)\right]
$$

and added to the systematic displacement $\Delta x_{\mathrm{F}}^{\mathrm{j}}$, yielding the total displacement $\Delta x^{j}$. As previously, the $x^{j}$ coordinate is updated and each trajectory is propagated until $x^{j} \geq L$.

Axial diffusion greatly broadens $t_{\text {res }}$ distributions for all CVs (Figure 2b). All $t_{\text {res }}$ still remain shorter than $0.2 \mathrm{~s}$, and ions start exiting FAIMS in just half that time. Charge density fluctuations would cause random displacements $\Delta x_{C}^{j}$ by Coulomb repulsion and thus further broadening. Since ions residing in FAIMS longer are more likely to be lost, broadening of $t_{\text {res }}$ distribution (by any mechanism) results in lower mean $t_{\text {res }}$ at any CV, but particularly near the peak baseline (Figure 2c). This phenomenon moderates the variation of $t_{\text {res }}$ as a function of CV (Figure 2c) and its already minor effect on $\mathrm{CV}$ spectral profiles (Figure 3).

In summary, the nonuniformity of gas flow velocity distribution and axial ion diffusion in FAIMS do not substantially affect CV spectral peak shapes. This is not to say that the distribution of residence times in FAIMS is unimportant. For fast scans, where the CV appreciably changes during ion transit through the device, the maximum scan rates and analytical response are controlled by $t_{\text {res }}$ distributions, a subject of ongoing research. Another conclusion is that, other factors being equal, the longitudinal field driven FAIMS [36, 37] (where the buffer gas is static) would perform very closely to conventional FAIMS, though the resolution might be marginally degraded.

\section{Realistic Asymmetric Waveform Profiles}

The other critical aspect of realistic FAIMS operation is the imperfection of asymmetric waveform. All previous FAIMS simulations assumed ideal $V_{\mathrm{D}}(t)$ functions, but real waveforms always carry electronic noise. This noise may have diverse physical origins, including thermal, shot, and inductive coupling of AC power and environmental RF. Many common sources produce "white" noise that has a uniform power spectrum. Some noises may be "pink" (that is, concentrated in certain frequency ranges) or have specific frequencies, for exam-



Figure 4. A segment of noisy bisinusoidal waveform $(f=2)$ synthesized by blending in simulated white noise (at $1.5 \%$ of $\left.V_{\text {max }}\right)$.

ple overtones of harmonics comprising the FAIMS waveform. The following modeling assumes white noise, which provides an unbiased way to gauge the overall impact of noise on FAIMS operation without delving into specific hardware details. The noise at extremely high frequencies ceases to have effect because ions do not have enough time to respond to variations of the field. Based on estimated relaxation times [38], those frequencies would exceed $\sim 0.1-1 \mathrm{GHz}$, depending on the ion and buffer gas. With this in mind, here we create pseudo-white noise $V_{\mathrm{N}}(t)$ by numerically blending $k_{\max }=1.5 \times 10^{5}$ phase-uncorrelated harmonics of equal intensity that cover the $0-4.2 \mathrm{MHz}$ frequency range:

$$
V_{\mathrm{N}}(t)=A_{\mathrm{N}} \sum_{k=1}^{k_{\max }} \sin \left(k w_{\Delta} t+2 \pi \chi_{k}\right)
$$

where $w_{\Delta}$ is the frequency interval (here $28 \mathrm{~Hz}$ ) and $\chi_{k}$ are random numbers between 0 and 1 . The resulting transient is scaled to a desired mean amplitude (below expressed as a percentage of $V_{\max }$ ) by adjusting $A_{\mathrm{N}}$ and superposed on the ideal $V_{\mathrm{D}}(t)$ (see Figure 4 ), then simulations are run as usual. As expected for a sufficiently white noise, other choices of $w_{\Delta}$ and/or $k_{\max }$ have produced the same outcome of FAIMS simulations.

The ripple is created by superposing on the ideal $V_{\mathrm{D}}(t)$ a harmonic $V_{\mathrm{R}}(t)=A_{\mathrm{R}} \sin \left(2 \pi w_{\mathrm{R}} t\right)$, where $w_{\mathrm{R}}$ and $A_{\mathrm{R}}$ are, respectively, the frequency and peak amplitude. Both noise and ripple may be imposed by adding $V_{\mathrm{N}}(t)$ and $V_{\mathrm{R}}(t)$. To fully understand the effect of ripple, we model FAIMS performance over a possible range of $w_{\mathrm{R}}$. The lowest practical $w_{\mathrm{R}}$ is determined by the ion residence time in FAIMS, and present $t_{\text {res }} \sim 0.2 \mathrm{~s}$ sets the limit of $w_{\mathrm{R}} \sim 20 \mathrm{~Hz}$, when the separation comprises about four ripple periods only. The other limit is defined by the requirement of ripple being a very slow modulation of the asymmetric waveform, which caps $w_{\mathrm{R}}$ at several $\mathrm{kHz}$. Here we consider $w_{\mathrm{R}}$ of 20,60 , and $600 \mathrm{~Hz}$ that approximately cover the reasonable range for present FAIMS conditions; the examples below are 


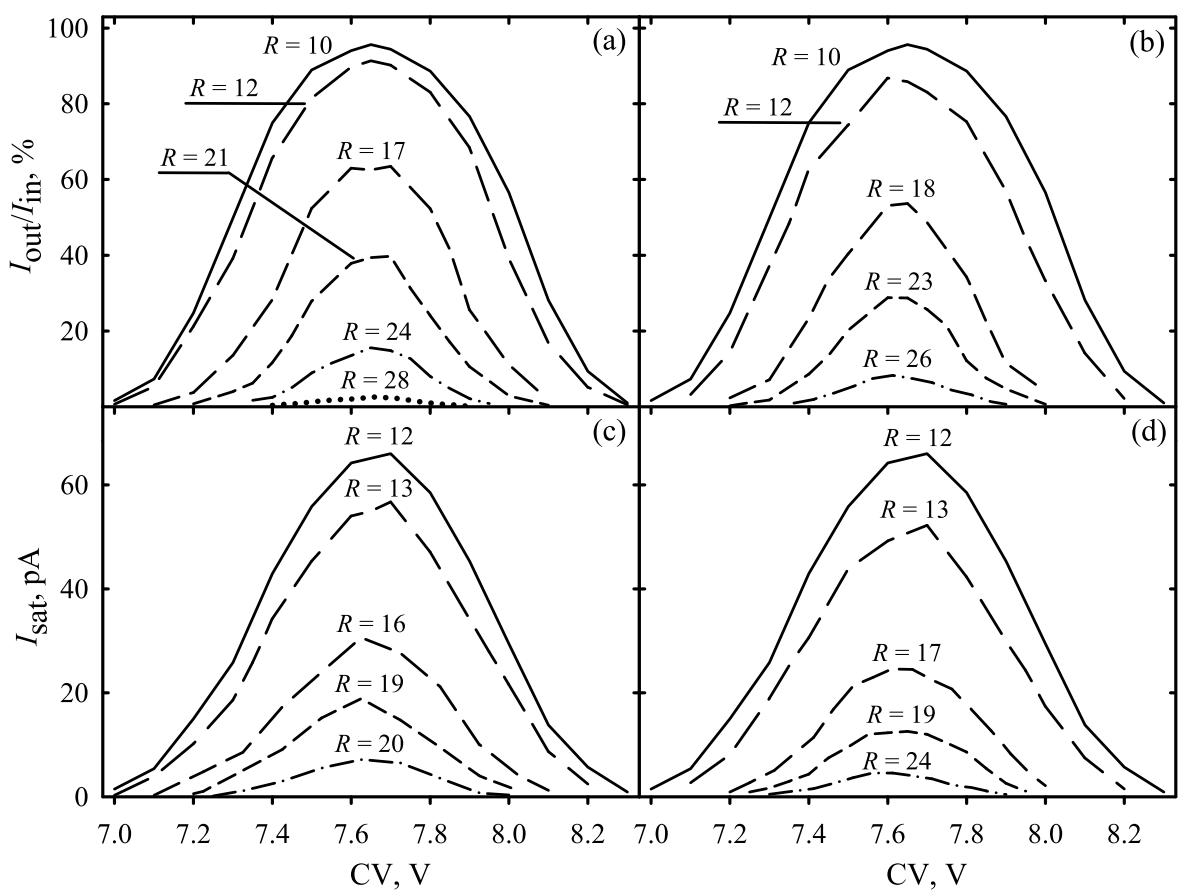

Figure 5. Dependence of CV spectral peaks on the level of noise (left panels) or ripple (right panels). The (Leucine $-\mathrm{H})^{-}$peak is simulated for the limits of zero and saturated ion current (top and bottom panels, respectively). In (a) and (c), the noise is at 0 (solid line), $1.0 \%$ (long dash), $2.0 \%$ (medium dash), $2.5 \%$ (short dash), $3.0 \%$ (dash-dot), and 3.5\% (dotted). In (b) and (d), $A_{\mathrm{R}}$ is 0 (solid line), $0.5 \mathrm{~V}$ (long dash), $1 \mathrm{~V}$ (medium dash), $1.25 \mathrm{~V}$ (short dash), and $1.5 \mathrm{~V}$ (dash-dot). Computed resolving power values are given for all features.

for $w_{\mathrm{R}}=60 \mathrm{~Hz}$ unless stated otherwise. The values for other FAIMS analyzers may differ; in particular, micromachined planar devices [23, 48] would require a significantly higher $w_{\mathrm{R}}$ range because of shorter $t_{\text {res }}$ and higher $w_{\mathrm{c}}$ in those systems. For example, $t_{\text {res }} \sim 2 \mathrm{~ms}$ means $w_{\mathrm{R}}>\sim 2 \mathrm{kHz}$.

\section{Influence of Noise and Ripple on FAIMS Response and Comparison with Experiments}

Simulations show that white noise of $\sim 1 \%$ and above strongly impacts FAIMS response, not surprising considering that the CVs of most analytes in standard operational regimes are $<1 \%$ of $V_{\max }$. For example, (Leucine $-\mathrm{H})^{-}$at $V_{\max }=-3.3 \mathrm{kV}$ has $\mathrm{CV}=7.7 \mathrm{~V}=$ $0.23 \%$ of $\pm V_{\max }$. Noise induces random motion of ion clouds in the gap perpendicularly to the gas flow. That disproportionately destroys ions located closer to electrodes, thus narrowing the $\mathrm{CV}$ spectral peaks and improving resolution at the expense of sensitivity (Figure 5a): the transmission efficiency (the ratio of ion current $I_{\text {out }}$ exiting FAIMS to incoming current $I_{\text {in }}$ ) decreases and eventually the gap becomes impassable (in this example, at $\sim 4 \%$ noise level).

The ripple that effectively narrows the gap produces a similar effect (Figure 5b), but at very low voltages. For example, with $w_{\mathrm{r}}=60 \mathrm{~Hz}$ the effect is significant already at $A_{\mathrm{R}} \sim 0.5 \mathrm{~V}$, i.e., just $0.015 \%$ of $\pm V_{\max }$. These voltages are $\sim 100$ times lower than those for white noise; for example the peak shapes with noise at 1 and $3 \%$ (i.e., mean voltages of 33 and $99 \mathrm{~V}$ ) in Figure $5 \mathrm{a}$ are close to those obtained with ripple at mean voltages (equal to $0.5 A_{\mathrm{R}}$ ) of 0.25 and $0.75 \mathrm{~V}$, respectively, in Figure $5 \mathrm{~b}$. This happens because high-frequency noise causes only local "wobbles" of ion trajectories that do not affect FAIMS separation substantially, and the fraction of low frequencies in white noise is small. The results naturally depend on $w_{R}$ : at lower frequencies the same effect is achieved with yet lower ripple amplitude while at higher $w_{\mathrm{R}}$ higher $A_{\mathrm{R}}$ are needed. For example, a peak close to that shown in Figure $5 \mathrm{~b}$ for $A_{\mathrm{R}}=1.0 \mathrm{~V}$ and $w_{\mathrm{R}}=60 \mathrm{~Hz}$ is generated at only $A_{\mathrm{R}} \sim 0.6 \mathrm{~V}$ when $w_{\mathrm{r}}=20 \mathrm{~Hz}$, but requires $A_{\mathrm{R}} \sim 8 \mathrm{~V}$ when $w_{\mathrm{R}}=600 \mathrm{~Hz}$ (not shown).

The behavior in the limit of saturated ion current is similar: both noise and ripple improve resolution but decrease the saturation current (Figure $5 c, d$ ). However, at high levels of noise (or ripple), a stronger Coulomb repulsion at higher ion currents appears to broaden $\mathrm{CV}$ spectral peaks rather than narrow them as for an ideal waveform [38]. The peak shapes are nearly independent of the ion current over a broad range of intermediate noise (ripple) levels; in the example of Figure 5 roughly $1.2-2.5 \%$ noise or $0.5-1.2 \mathrm{~V}$ ripple. This is demonstrated more clearly by plotting peaks computed at a fixed noise (ripple) level in the limits of zero and saturated ion current (Figure 6a and b).

No significant dependence of $\mathrm{CV}$ spectral peak 

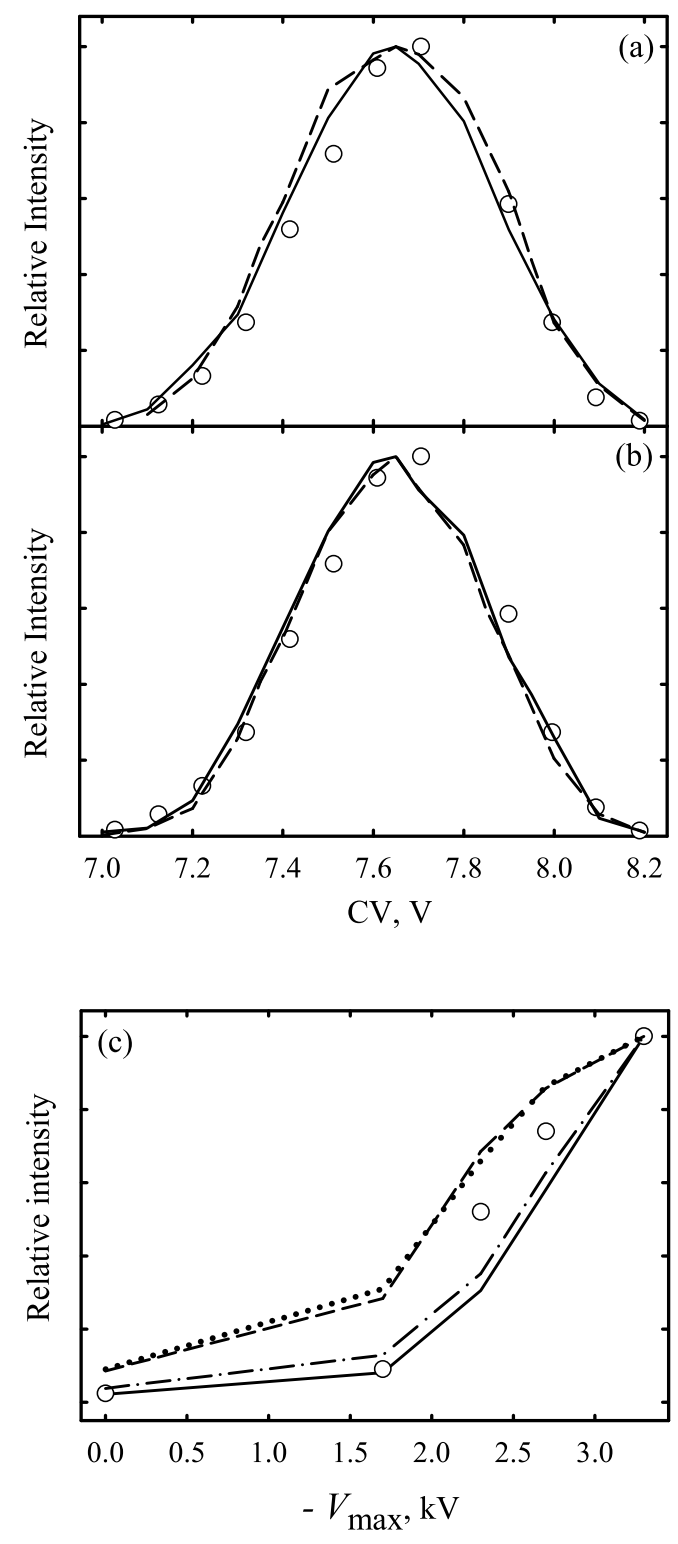

Figure 6. Calculations (lines) and measurements [49] (circles) for (Leucine $-\mathrm{H})^{-}$. (a) and (b) are CV spectral peak profiles with (a) noise at $1.5 \%$ or (b) ripple at $A_{\mathrm{R}}=0.75 \mathrm{~V}$; dashed and solid lines are, respectively, for zero and saturated ion current limits. In (c) is sensitivity as a function of dispersion voltage; dashed and solid lines are for zero and saturated current limits without noise; dotted and dash-dot are for the same with noise at $1.5 \%$.

shapes on ion signal intensity in FAIMS (for example, controlled by analyte concentration) has been reported. This appears to contravene earlier calculations (for perfect asymmetric waveforms) where peaks narrowed at higher ion currents [38]. Present modeling would remove this discrepancy, if the imperfections of waveforms in existing FAIMS devices fall within the ballpark where the resolution depends only weakly on ion current. Further, measured peaks are generally narrower and sharper than simulations for low ion current and could previously be fit only by assuming conditions close to the saturated-current limit that is often not realistic [38, 39]. Present simulations at modest noise or ripple levels (for any ion current) match the CV peak profiles obtained using Selectra and similar cylindrical analyzers reasonably well (Figure 6a, b).

The sensitivity of cylindrical FAIMS increases at higher dispersion voltages (up to a point) because of ion focusing. Modeling for perfect waveforms has fully reproduced this effect $[38,39]$. We do not find the noise or ripple to influence this behavior significantly at any ion current from zero limit to saturated conditions; in other words the sensitivity decreases proportionately at all $V_{\max }$ (Figure 6c). So the agreement between simulations and measurements for FAIMS ion focusing is retained for reasonably noisy waveforms.

\section{General FAIMS Resolution-Sensitivity Curve}

As has been discussed [39], FAIMS resolution could be increased at the expense of sensitivity by decreasing the gap width and/or asymmetric waveform frequency, or (in certain cases) changing the buffer gas. All three methods produce equivalent results at any ion current [39]. The curves defining the resolution-sensitivity trade-off for zero- and saturated-current limits are plotted in Figure 7. Adjusting the amplitude of noise or ripple produces a trade-off that is similar, but in general

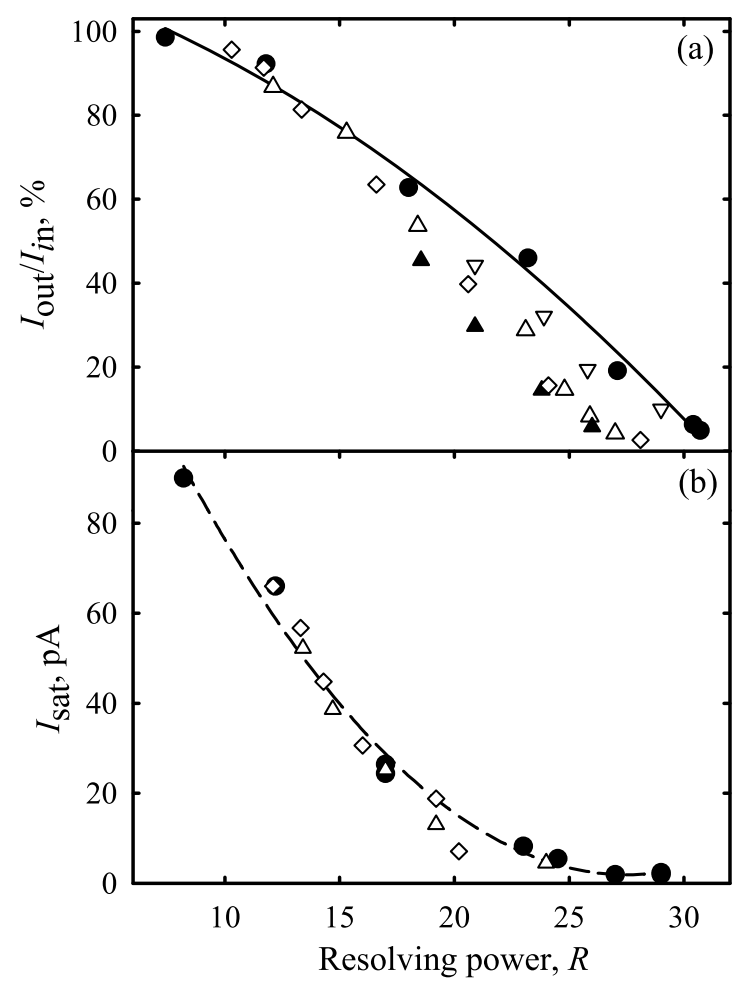

Figure 7. Resolution-sensitivity curves for (Leucine $-\mathrm{H})^{-}$, in the limits of zero (a) and saturated (b) ion current. Symbols stand for various means of resolution control: white noise (diamonds), ripple (triangles), and adjusting the gap width and/or waveform frequency (circles) [39]; lines are second-order regressions through the circles. Ripple frequencies are: $600 \mathrm{~Hz}$ (open inverted triangles), $60 \mathrm{~Hz}$ (open triangles), and $20 \mathrm{~Hz}$ (filled triangles). 
a bit inferior (Figure 7). Increasing the perturbation frequency is beneficial: the performance is very close to the ideal limit using a $600 \mathrm{~Hz}$ ripple, but significantly worse at $w_{\mathrm{r}}=60 \mathrm{~Hz}$ and especially $20 \mathrm{~Hz}$ (Figure 7a). Of course, the curves in Figure 7 are specific to (Leucine $\mathrm{H})^{-}$and those for other analytes differ. In particular, species with stronger $K(E)$ dependence allow a higher resolving power (often 2 to 3 times that in Figure 7) at equal sensitivity [39].

Although apparently optimum on the fundamental level, varying the gap width and/or waveform frequency [39] is cumbersome in practice: the first requires mechanical adjustments and would also change the gas flow velocity (and thus the ion residence time) and CV values, while the second involves major electrical hardware changes and has limited range. In contrast, the ripple amplitude has no bearing on measured $\mathrm{CVs}$, ion residence times, or gas flow, and can be rapidly and flexibly controlled on a software level during analyses.

\section{Conclusions}

We have extended the simulation of FAIMS operation beyond an idealized one-dimensional picture with uniform gas flow and perfect waveforms. The improved model incorporates a realistic distribution of gas flow velocities and axial diffusion, and accounts for inevitable perturbations of asymmetric waveform, whether intentional or a manifestation of electronic noise. The nonuniformity of gas flow velocity profile and axial ion diffusion in the analytical gap have only a minor effect on FAIMS separations at static instrumental conditions (i.e., constant $\mathrm{CV}$ ), slightly improving resolution. This means that the longitudinal field driven and conventional FAIMS devices would be very close in performance.

The effect of waveform perturbations is inversely correlated with frequency, and at low frequencies may be significant even for background noise at very low levels $(\sim 0.01 \%$ of nominal voltage). This is the case (unlike for quadrupole MS or IMS separations) because FAIMS is based on a small difference of two large quantities and thus is highly sensitive to minor variations of either, and because long ion residence times permit minute perturbations to accumulate. Electronic noise at either random frequencies (white noise) or fixed frequency (ripple) always decreases sensitivity but, somewhat counter-intuitively, improves resolution. This is due to the nature of FAIMS as a filter: perturbing the ion trajectories necessarily reduces the stability region in the gap and thus narrows the range of conditions (i.e., CV) allowing ion transmission. Variation of noise or ripple amplitude allows a control of the trade-off between FAIMS resolution and sensitivity, though somewhat less effective on a physical level than that by adjustment of gap width and/or asymmetric waveform frequency. However, that disadvantage diminishes and eventually almost vanishes with increasing ripple frequency. Thus, from an instrumental per- spective, scaling of the ripple amplitude at sufficiently high frequency appears as the most sensible approach to FAIMS resolution control. Making resolution tunable via ripple control would substantially expand the practical utility of FAIMS analyzers.

\section{Acknowledgments}

The authors are grateful to Dr. R. Guevremont, Dr. R. Purves, and colleagues (Ionalytics Corporation), Professor J. de la Mora (Yale University), D. Prior, G. Anderson, and Dr. F. Li (PNNL), and I. Krutova (FAU) for sharing unpublished results and for valuable discussions. They also thank the NIH National Center for Research Resources (RR18522) and the U.S. Department of Energy (PNNL Laboratory Directed Research and Development Program) for supporting portions of this research. Pacific Northwest National Laboratory is operated by Battelle for the DOE through contract DE-AC05-76RLO1830.

\section{References}

1. Gorshkov, M. P. USSR Inventor's Certificate, 1982; no. 966583.

2. Buryakov, I. A., Krylov, E. V., Soldatov, V. P. USSR Inventor's Certificate, 1987; no. 1,337,934.

3. Buryakov, I. A., Krylov, E. V., Soldatov, V. P. USSR Inventor's Certificate, 1988; no. 1,412,447.

4. Buryakov, I. A., Krylov, E. V., Soldatov, V. P. USSR Inventor's Certificate, 1989; no. 1,485,808.

5. Buryakov, I. A.; Krylov, E. V.; Makas, A. L.; Nazarov, E. G.; Pervukhin, V. V.; Rasulev, U. K. Ion Division by Their Mobility in High Tension Alternating Electric Field. Sov. Tech. Phys. Lett. 1991, 17, 446.

6. Buryakov, I. A.; Krylov, E. V.; Makas, A. L.; Nazarov, E. G.; Pervukhin, V. V.; Rasulev, U. K. Drift Spectrometer for the Control of Amine Traces in the Atmosphere. J. Anal. Chem. 1993, 48, 114-121.

7. Buryakov, I. A.; Krylov, E. V.; Nazarov, E. G.; Rasulev, U. K. A New Method of Separation of Multiatomic Ions by Mobility at Atmospheric Pressure Using a High-Frequency AmplitudeAsymmetric Strong Electric Field. Int. J. Mass Spectrom. Ion Processes 1993, 128, 143-148.

8. Guevremont, R.; Purves, R. W. Atmospheric Pressure Ion Focusing in a High-Field Asymmetric Waveform Ion Mobility Spectrometer. Rev. Sci. Instrum. 1999, 70, 1370-1383.

9. Guevremont, R.; Purves, R. W. Electrospray Ionization HighField Asymmetric Waveform Ion Mobility Spectrometry-Mass Spectrometry. Anal. Chem. 1999, 71, 2346-2357.

10. Ells, B.; Barnett, D. A.; Froese, K.; Purves, R. W.; Hrudey, S.; Guevremont, R. Detection of Chlorinated and Brominated Byproducts of Drinking Water Disinfection Using Electrospray Ionization-High-Field Asymmetric Waveform Ion Mobility Spectrometry-Mass Spectrometry. Anal. Chem. 1999, 71, 4747-4752.

11. Handy, R.; Barnett, D. A.; Purves, R. W.; Horlick, G.; Guevremont, R. Determination of Nanomolar Levels of Perchlorate in Water by ESI-FAIMS-MS. J. Anal. At. Spectrom. 2000, 15, 907-911.

12. Ells, B.; Froese, K.; Hrudey, S. E.; Purves, R. W.; Guevremont, R.; Barnett, D. A. Detection of Microcystins Using Electrospray Ionization High-Field Asymmetric Waveform Ion Mobility Spectrometry/Mass Spectrometry. Rapid Commun. Mass Spectrom. 2000, 14, 1538-1542.

13. Gabryelski, W.; Wu, F.; Froese, K. L. Comparison of HighField Asymmetric Waveform Ion Mobility Spectrometry with GC Methods in Analysis of Haloacetic Acids in Drinking Water. Anal. Chem. 2003, 75, 2478-2486. 
14. McCooeye, M. A.; Ells, B.; Barnett, D. A.; Purves, R. W.; Guevremont, R. Quantitation of Morphine and Codeine in Human Urine Using High-Field Asymmetric Waveform Ion Mobility Spectrometry (FAIMS) with Mass Spectrometric Detection. J. Anal. Toxicol. 2001, 25, 81-87.

15. McCooeye, M. A.; Mester, Z.; Ells, B.; Barnett, D. A.; Purves, R. W.; Guevremont, R. Quantitation of Amphetamine, Methamphetamine, and their Methylenedioxy Derivatives in Urine by Solid-Phase Microextraction Coupled with Electrospray Ionization-High-Field Asymmetric Waveform Ion Mobility Spectrometry-Mass Spectrometry. Anal. Chem. 2002, 74, 30713075.

16. McCooeye, M.; Ding, L.; Gardner, G. J.; Fraser, C. A.; Lam, J.; Sturgeon, R. E; Mester, Z. Separation and Quantitation of the Stereoisomers of Ephedra Alkaloids in Natural Health Products Using Flow Injection-Electrospray Ionization-High Field Asymmetric Waveform Ion Mobility Spectrometry-Mass Spectrometry. Anal. Chem. 2003, 75, 2538-2542.

17. Cui, M.; Ding, L.; Mester, Z. Separation of Cisplatin and Its Hydrolysis Products Using Electrospray Ionization High-Field Asymmetric Waveform Ion Mobility Spectrometry Coupled with Ion Trap Mass Spectrometry. Anal. Chem. 2003, 75, 5847-5853.

18. Gabryelski, W.; Froese, K. L. Rapid and Sensitive Differentiation of Anomers, Linkage, and Position Isomers of Disaccharides Using High-Field Asymmetric Waveform Ion Mobility Spectrometry (FAIMS). J. Am. Soc. Mass Spectrom. 2003, 14, 265-277.

19. Buryakov, I. A.; Kolomiets, Y. N.; Luppu, B. V. Detection of Explosive Vapors in the Air Using an Ion Drift Nonlinearity Spectrometer. J. Anal. Chem. 2001, 56, 336-340.

20. Buryakov, I. A.; Kolomiets, Y. N. Rapid Determination of Explosives and Narcotics Using a Multicapillary-Column Gas Chromatograph and an Ion-Mobility Spectrometer. J. Anal. Chem. 2003, 58, 944-950.

21. Buryakov, I. A. Qualitative Analysis of Trace Constituents by Ion Mobility Increment Spectrometer. Talanta 2003, 61, 369375.

22. Buryakov, I. A. Express Analysis of Explosives, Chemical Warfare Agents and Drugs with Multicapillary Column Gas Chromatography and Ion Mobility Increment Spectrometry. J. Chromatogr. B 2004, 800, 75-82.

23. Eiceman, G. A.; Krylov, E. V.; Krylova, N. S.; Nazarov, E. G.; Miller, R. A. Separation of Ions from Explosives in Differential Mobility Spectrometry by Vapor-Modified Drift Gas. Anal. Chem. 2004, 76, 4937-4944.

24. Guevremont, R. High-Field Asymmetric Waveform Ion Mobility Spectrometry: A New Tool for Mass Spectrometry. J. Chromatogr. A 2004, 1058, 3-19.

25. Veasey, C. A.; Thomas C. L. P. Fast Quantitative Characterization of Differential Mobility Responses. Analyst 2004, 129, 198-204.

26. Shvartsburg, A. A.; Hudgins, R. R.; Dugourd, P.; Jarrold, M. F. Structural Information from Ion Mobility Measurements: Applications to Semiconductor Clusters. Chem. Soc. Rev. 2001, 30, $26-35$.

27. Creaser, C. S.; Griffiths, J. R.; Bramwell, C. J.; Noreen, S.; Hill, C. A.; Thomas, C. L. P. Ion Mobility Spectrometry: A Review. Part 1. Structural Analysis by Mobility Measurement. Analyst 2004, 129, 984-994.

28. Purves, R. W. Guevremont, R.; Day, S.; Pipich, C. W.; Matyjaszczyk, M. S. Mass Spectrometric Characterization of a High-Field Asymmetric Waveform Ion Mobility Spectrometer. Rev. Sci. Instrum. 1998, 69, 4094-4105.

29. Guevremont, R.; Barnett, D. A.; Purves, R. W.; Viehland, L. A. Calculation of Ion Mobilities from Electrospray Ionization
High-Field Asymmetric Waveform Ion Mobility Spectrometry Mass Spectrometry. J. Chem. Phys. 2001, 114, 10270-10277.

30. Meek, J. M.; Craggs, J. D.; Eds.; Electrical Breakdown of Gases; Wiley: New York, 1978.

31. Lozeille, J.; Winata, E.; Soldan, P.; Lee, E. P. F.; Viehland, L. A.; Wright, T. G. Spectroscopy of $\mathrm{Li}^{+} \cdot \operatorname{Rg}$ and $\mathrm{Li}^{+}-\mathrm{Rg}$ Transport Coefficients $(\mathrm{Rg}=\mathrm{He}-\mathrm{Rn})$. Phys. Chem. Chem. Phys. 2002, 4 , 3601-3610.

32. Viehland, L. A.; Lozeille, J.; Soldan, P.; Lee, E. P. F.; Wright, T. G. Spectroscopy of $\mathrm{Na}^{+} \cdot \mathrm{Rg}$ and Transport Coefficients of $\mathrm{Na}^{+}$in $\mathrm{Rg}(\mathrm{Rg}=\mathrm{He}-\mathrm{Rn})$. J. Chem. Phys. 2003, 119, 3729-3736.

33. Barnett, D. A.; Ells, B.; Guevremont, R.; Purves, R. W.; Viehland, L. A. Evaluation of Carrier Gases for Use in High-Field Asymmetric Waveform Ion Mobility Spectrometry. J. Am. Soc. Mass Spectrom. 2000, 11, 1125-1133.

34. Thackston, M. G.; Eisele, F. L.; Ellis, H. W.; McDaniel, E. W. Mobilities of $\mathrm{Cs}^{+}$Ions in Molecular Gases $\mathrm{H}_{2}, \mathrm{~N}_{2}, \mathrm{O}_{2}, \mathrm{CO}$, and $\mathrm{CO}_{2}$. J. Chem. Phys. 1977, 67, 1276-1277.

35. Pope, W. M.; Ellis, H. W.; Eisele, F. L.; Thackston, M. G.; McDaniel, E. W.; Langley, R. A. Mobilities and Longitudinal Diffusion Coefficients for $\mathrm{Cs}^{+}$ions in He and Ne Gas. J. Chem. Phys. 1978, 68, 4761-4762.

36. Miller, R. A.; Zahn, M. Longitudinal Field Driven Field Asymmetric Ion Mobility Filter and Detection System. US Patent, 2003; no. 6,512,224

37. Miller, R. A.; Zahn, M. Longitudinal Field Driven Ion Mobility Filter and Detection System. US Patent, 2004; No. 6,815,669.

38. Shvartsburg, A. A.; Tang, K.; Smith, R. D. Modeling the Resolution and Sensitivity of FAIMS Analyses. J. Am. Soc. Mass Spectrom. 2004, 15, 1487-1498.

39. Shvartsburg, A. A.; Tang, K.; Smith, R. D. Optimization of the Design and Operation of FAIMS Analyzers. J. Am. Soc. Mass Spectrom. 2005, 16, 2-12.

40. Carnahan, B. L.; Tarassov, A. S. Ion Mobility Spectrometer. US Patent, 1995; no. 5,420,424

41. Shvartsburg, A. A.; Tang, K.; Smith, R. D. Understanding and Designing Field Asymmetric Waveform Ion Mobility Spectrometry Separations in Gas Mixtures. Anal. Chem. 2004, 76, 7366-7374.

42. Ells, B.; Barnett, D. A.; Purves, R. W.; Guevremont, R. Detection of Nine Chlorinated and Brominated Haloacetic Acids at Part-per-Trillion Levels Using ESI-FAIMS-MS. Anal. Chem. 2000, 72, 4555-4559.

43. Guevremont, R.; Barnett, D. A.; Purves, R. W.; Vandermey, J. Analysis of a Tryptic Digest of Pig Hemoglobin Using ESIFAIMS-MS. Anal. Chem. 2000, 72, 4577-4584.

44. Buryakov, I. A. Coefficient of Ion Mobility Versus Electric Field Strength Dependence in Gases: Experimental Determination. Tech. Phys. 2002, 47, 1453-1457.

45. Buryakov, I. A. Determination of Kinetic Transport Coefficients for Ions in Air as Functions of Electric Field and Temperature. Tech. Phys. 2004, 49, 967-972.

46. Schlichting, H. Boundary-Layer Theory; McGraw-Hill: New York, 1979, pp 185-187, 241.

47. Elistratov, A. A.; Shibkov, S. V. An Analysis of Nonlinear Ion Drift Spectrometry for Gas Detectors with Separating Chamber of Planar Geometry. Tech. Phys. Lett. 2003, 29, 81-83.

48. Miller, R. A.; Nazarov, E. G.; Eiceman, G. A.; King, A. T. A MEMS Radio-Frequency Ion Mobility Spectrometer for Chemical Vapor Detection. Sens. Actuators A 2001, 91, 301-312.

49. Barnett, D. A.; Ells, B.; Guevremont, R.; Purves, R. W. Separation of Leucine and Isoleucine by Electrospray IonizationHigh Field Asymmetric Waveform Ion Mobility SpectrometryMass Spectrometry. J. Am. Soc. Mass Spectrom. 1999, 10, 1279 1284. 